REVISTA PROYECCIONES No $10: 135-137$

Diciembre 1985 - ISSN 0716-0917

Jornada Matemáticas, Agosto 1985.

\title{
UNA EXTENSIÓN DEL TEOREMA DE WARNER
}

Dr. SAMUEL NAVARRO\%

Sea X espacio topológico ultraregular, (F, ||) un cuerpo valuado no trivial, esféricamente completo.

$6(\mathrm{X}, \mathrm{F})$ denota el espacio de funciones escalares definidas en $\mathrm{X}$, provisto de la topología de la convergencia uniforme sobre los compactos de X. (Ver Survey sobre esta topología en Navarro (3)).

E1 espacio $\sigma(X, F)$ es completo si $(F, \|)$ es un cuerpo local (Bourbaki (1)).

Observando la demostración de la extensión del teorema de Tietze al caso no arquimedeano R. El1is (2) se puede deducir que toda función f definida y continua en un subconjunto compacto $\mathrm{K}$ de un espacio ultraregular $\mathrm{X}$ tiene una extensión a todo el espacio $\mathrm{X}$, acotada por la misma constante que acota $f$ en $K$.

Con esta observación podemos enunciar una extensión del teorema de caracterización de la completitud de Wagner (4) para el espacio de funciones continuas.

* Departamento de Matemáticas, Universidad de Santiago, Santiago. 
Diremos que un espacio ultraregular $\mathrm{X}$ es $\mathrm{X}_{\mathrm{F}}$-espacio si todo elemento de $(X ; F)$ cuya restricción a cada compacto $K \bar{c} \mathrm{~K}$ es continua, pertenece a $\delta(X ; F)$; recordamos que un e.v.t. es casi-completo si todo cerrado acotado es completo.

\section{TEOREMA:}

Sea (F, ||) un cuerpo local no trivial, entonces las siguientes proposiciones son equivalentes.
1) $\boldsymbol{f}(\mathrm{X} ; \mathrm{F})$ es completo
2) $6(X ; F)$ es casi-completo
3) $\mathrm{X}$ es $k_{F}$-espacio.

DEMOSTRACION :

$$
\begin{aligned}
& \text { 1) } \Rightarrow 2) \text { es claro } \\
& \text { 2) } \Rightarrow 3) \text { supongamos que } f \in \mathscr{F}(\mathrm{X} ; \mathrm{F}) \text { es acotada. Llamamos }
\end{aligned}
$$
$f_{k}$ la restricción de $f$ a $K$; $K$ compacto. $S 1 f_{k} G 6(K ; F)$ entonces existe $\hat{f}_{k}$, extensión de $f_{k}$ a $X$ tal que

$$
\operatorname{Sup}_{X} \hat{f}_{k}(x)<\operatorname{Sup}_{X} f(x)
$$

Sean $K$ el conjunto de los compactos de $\mathrm{x}$ y $\mathscr{F}$ el filtro inducido por la red trivial que se forma en $K$.

Consideramos

$$
\alpha: \mathcal{X} \Rightarrow 6(\mathrm{X} ; \mathrm{F})
$$

tal que $(K)=\hat{\mathrm{f}}_{\mathrm{k}} \quad \mathrm{y}$ 


$$
A=\left\{h \in G(X ; F) / \operatorname{Sup}_{X}|h(x)|<\operatorname{Sup}_{X}|\dot{f}(x)|\right\}
$$

Entonces $\alpha(\mathbb{F})$ es una base de filtro en A y el filtro generado

G $\alpha(\widetilde{\sigma})$ es un filtro convergente a $f$ en $\mathscr{F}(X ; F)$, en efecto, sea

$f+M(K ; U)$ una vecindad de $f$ en $\mathscr{f}(X, F)$ entonces el conjunto $B_{H}=\left\{\hat{\mathrm{f}}_{\mathrm{S}} / \mathrm{H} \subseteq \mathrm{S}, \mathrm{S}\right.$ compacto $\}$

pertenece a $\alpha(\mathscr{F}), y$

$$
\mathrm{B}_{\mathrm{H}} \subseteq \mathrm{f}+\mathrm{M}(\mathrm{H} ; \mathrm{U})
$$

Luego

$$
f+M(H ; U) \in \widetilde{F}_{\alpha(\mathcal{F})}
$$

Por otra parte $A$ es un conjunto cerrado y acotado en $6(X ; F)$, lue go completo, de donde se obtiene que $A$ es cerrado en $F(X, F)$. Finalmente obtenemos que $f \in \mathrm{A}$.

Para el caso general, suponemos que $\mathrm{f} \in \mathbb{F}(\mathrm{X}, \mathrm{F})$ y que cada restric ción a los compactos de $\mathrm{X}$ es continua. Escogemos $\left(\alpha_{n}\right)$ una sucesión en $F$ de valores absolutos estrictamente creciente. Consideramos las funciones $\mathrm{f}_{\mathrm{n}}$ definida por

$$
f_{n}(X)= \begin{cases}f(x) & \text { si }\left|f_{n}(x)\right|<\alpha_{n} \\ \alpha_{n} & \text { si }\left|f_{n}(x)\right|>\alpha_{n}\end{cases}
$$

cada $f_{n}$ es acotada y su restricción a un compacto es continua, luego $\overline{\mathrm{f}}_{\mathrm{n}} \in \mathcal{G}(\mathrm{X}, \mathrm{F}) \quad \forall \mathrm{n}$.

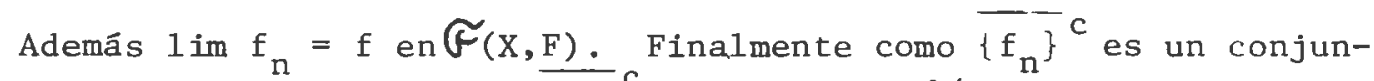
to completo en $6(X, F)$, resulta que $\overline{\left\{f_{n}\right\}^{c}}$ es cerrado en $\widetilde{F}(X ; F)$ de donde se tiene que $f \in 6(X ; F)$. La implicación $c) \Rightarrow$ a) sigue consideraciones genera les (Ver Bourbaki (1)). 